OC from MTX-treated patients, the basal expression of key genes encoding TRAP, integrin b3 and cathepsin $\mathrm{K}$ was higher than in patients under biological therapy. No difference was observed when comparing anti-TNF and tocilizumab-treated patients. The expression of OC-specific genes in monocytes correlated with disease activity $(\mathrm{p}<0.05)$.

Conclusion Anti-TNF and tocilizumab-treated patients have a reduced expression of key genes in OC and monocytes. OC-specific gene expression on monocytes correlates with disease activity, suggesting that the expression of genes that leads to differentiation in $\mathrm{OC}$ is regulated by the inflammatory environment.

\section{A50 INFLUENCE OF ANTI-TUMOUR NECROSIS FACTOR DRUGS, TOCILIZUMAB AND METHOTREXATE ON OSTEOCLAST DIFFERENTIATION}

I Perpétuo, ${ }^{1} \mathrm{~J}$ Caetano-Lopes, ${ }^{1}$ A Rodrigues, ${ }^{1,2}$ J Polido-Pereira, ${ }^{1,2}$ M Viana Queiroz, ${ }^{2}$ J E Fonseca, ${ }^{1,2}$ H Canhão1, 2 'Unidade de Investigação em Reumatologia, Instituto de Medicina Molecular/Faculdade de Medicina de Lisboa; ${ }^{2}$ Rheumatology and Bone Metabolic Diseases Department, Hospital de Santa Maria, Lisboa, Portugal

\subsection{6/ard.2010.129593}

Background Rheumatoid arthritis (RA) is a systemic disease characterised by hyperactivation of the immune system and by stimulation of bone resorption by activation of osteoclasts (OC) leading to joint destruction.

Objective To assess the activity of OC differentiated from circulating monocytes in patients with RA treated with methotrexate (MTX), anti-tumour necrosis factor (anti-TNF) drugs and tocilizumab.

Patients and Methods 19 women with RA were recruited, 6 of whom were being treated with MTX, 7 with anti-TNF drugs and 6 with tocilizumab. Monocytes were isolated from blood and cultured for 25 days in the presence of macrophage colony-stimulating factor, receptor activator for nuclear factor $\mathrm{\kappa B}$ ligand, dexamethasone and transforming growth factor $\beta$. Tartrate-resistant acid phosphatase (TRAP) staining and resorption assays were performed on day 21 of culture. OC-specific surface $\alpha$ versus $\beta(3)$ integrin was detected by flow cytometry at days 14, 21 and 25 of culture. Expression of OC-specific genes was assessed in monocytes and in OC on day 21 of culture by real-time PCR.

Results The disease activity score using 28 joint counts (DAS28) was 3.55 in patients treated with biological agents and 4.97 in those treated with MTX. Disease activity was significantly lower in the group treated with biological agents $(p<0.05)$. The statistical analysis was corrected for the differences in disease activity. Anti-TNF-treated patients had a delay in $\alpha$ versus $\beta(3)$ integrin-positive cell (pre-OC and OC) formation. Moreover, it was found that, in monocytes and 